\title{
Percepção de Agentes Comunitários de Saúde sobre uma Formação em Desenvolvimento Infantil e Indicadores de Risco
}

\author{
Georgius Cardoso Esswein ${ }^{1}$ \\ ${ }^{1}$ Universidade Federal do Rio Grande do Sul, RS, Brasil. \\ Aline Facchin Rovaris ${ }^{2}$ \\ ${ }^{2}$ Centro de Atenção Psicossocial Novos Tempos, RS, Brasil.
}

\author{
Gabrielli Pohlmann Rocha ${ }^{3}$ \\ ${ }^{3}$ Universidade Estadual de Campinas, SP, Brasil. \\ Daniela Centenaro Levandowski ${ }^{4}$ \\ ${ }^{4}$ Universidade Federal de Ciência da Saúde \\ de Porto Alegre, RS, Brasil.
}

Resumo: Esse estudo objetivou descrever a percepção de agentes comunitários de saúde (ACS) de Porto Alegre acerca de uma formação sobre desenvolvimento infantil e indicadores de risco. Essa formação visou a instrumentalização dos ACS para o uso do IRDI (Indicadores Clínicos de Risco para o Desenvolvimento Infantil) e foi organizada em dois módulos: teórico e prático. Foram realizados grupos focais após cada módulo para avaliar a percepção dos ACS, e a análise temática dos relatos dos grupos focais originou três temas: metodologia da formação, dificuldades encontradas e repercussões da formação. Os participantes mencionaram a articulação teoriaprática e a acessibilidade da linguagem como recursos que auxiliaram na compreensão dos temas abordados. Mudanças na compreensão sobre o desenvolvimento infantil e qualificação do olhar sobre a infância foram as principais repercussões da formação sobre o trabalho dos ACS. As dificuldades se centraram na complexidade do IRDI e nas condições de trabalho para o seu uso. O estudo demonstrou a adequação da estrutura e do conteúdo da formação, bem como a possibilidade de uso do IRDI como estratégia de cuidado dos ACS na Atenção Básica.

Palavras-chave: Atenção Primária à Saúde, Agente Comunitário de Saúde, Educação Continuada, Desenvolvimento Infantil.

\section{Perceptions of Community Health Agents about a course on child development and risk indicators}

\begin{abstract}
This study aimed to describe the perception of Community Health Agents (CHA) from Porto Alegre about a course on child development and riskindicators. This course was organized into a practical and a theoretical module, seeking to prepare CHA for the use of risk indicators for child development (RICD). After each module, CHA perception was assessed by focus groups. Groups reports underwent thematic analysis, generating three themes: training methodology, difficulties, and training repercussions. According to the participants, the articulation between theory-practice and language accessibility helped them to understand the topics covered throughout the course. The main repercussions of the course on the work of CHA included changes in understanding regarding child development and the qualification of perspectives on childhood. Participants also reported difficulties regarding the complexity of RICD and the workplace conditions for its use. This study demonstrates the adequacy of the course structure and content, as well as the possibility of CHA using the RICD as a care strategy in Primary Health Care.
\end{abstract}

Keywords: Primary Health Care, Community Health Agents, Continuing Education, Child Development. 


\title{
Percepción de Agentes Comunitarios de Salud sobre la capacitación en desarrollo infantil e indicadores de riesgo
}

\begin{abstract}
Resumen: Este estudio tuvo como objetivo describir la percepción de Agentes Comunitarios de Salud (ACS) de Porto Alegre sobre la capacitación en desarrollo infantil e indicadores de riesgo. La propuesta pretendió instrumentalizarlos para el uso de IRDI (Indicadores Clínicos de Riesgo para el Desarrollo Infantil) y constó de dos módulos: teórico y práctico. Se realizaron grupos focales después de cada módulo para evaluar la percepción de los ACS. Del análisis temático de los relatos de los grupos focales emergieron tres temas: metodología de la capacitación, dificultades encontradas y repercusiones de la capacitación. Los participantes mencionaron la articulación teoría-práctica y la accesibilidad del lenguaje como los recursos que les ayudaron en la comprensión de los temas abordados. Los cambios en la comprensión del desarrollo infantil y la calificación sobre la forma de entender la infancia fueron las principales repercusiones de la capacitación sobre el trabajo de los ACS. Las dificultades se centraron en la complejidad de IRDI y en las condiciones laborales para su uso. El estudio demostró la adecuación de la estructura y del contenido de la capacitación, así como la posibilidad de uso de IRDI como estrategia de cuidado de los ACS en la Atención Primaria de Salud.
\end{abstract}

Palabras clave: Atención Primaria de Salud, Agentes Comunitarios de Salud, Educación Continua, Desarrollo Infantil.

\section{Introdução}

A Atenção Básica (AB) é reconhecida como a "porta de entrada" para a atenção em saúde no Brasil. Caracteriza-se como um conjunto de ações em âmbito individual e coletivo, cujos níveis de atuação englobam promoção, prevenção, diagnóstico, tratamento e reabilitação em saúde. Atualmente, a Estratégia de Saúde da Família (ESF) é sua maior expressão.

A ESF tem uma posição privilegiada no acompanhamento de famílias e identificação das demandas em saúde e conta com equipes multiprofissionais (Ministério da Saúde, 2006, 2012, 2017). Um dos principais profissionais que atuam nessa estratégia é o Agente Comunitário de Saúde (ACS), categoria implementada no Sistema Único de Saúde (SUS) em 1991, por meio do Programa de Agentes Comunitários de Saúde (PACS) (Bachilli, Sacavassa, \& Spiri, 2008). Dentre as suas principais atribuições estão o cadastro e a orientação das famílias em relação aos serviços de saúde, bem como seu acompanhamento por meio de visita domiciliar; realização de ações de integração unidade-território; e o desenvolvimento de ações programadas e de demanda espontânea de prevenção e promoção de saúde (Ministério da Saúde, 2012, 2017).
Por residir na comunidade em que trabalha, o ACS é um profissional em posição de mão dupla, uma vez que interrelaciona o saber técnico das equipes aos saberes populares (Riquinho, Pellini, Ramos, Silveira, \& Santos, 2017). Portanto, é um elemento que tem uma função importante na promoção de saúde, por meio de estratégias educativas e da identificação das demandas do território e consolidação do SUS (Ministério da Saúde, 2009a, 2012, 2017; Morosini, Corbo, \& Guimarães, 2007).

Um dos eixos relevantes de atuação dos ACS é a saúde da criança, por meio de diversas atividades que abarcam a identificação de fatores de risco à saúde, violência e deficiência; o incentivo ao cumprimento do calendário vacinal; a busca ativa; orientações às famílias, dentre outras. O ACS se destaca também na prática de acompanhamento do desenvolvimento infantil. Neste sentido, esses profissionais podem ajudar na identificação de sinais de risco ainda nos anos iniciais (Ministério da Saúde, 2009a, 2009b).

No que tange ao desenvolvimento infantil, embora desenvolvimento seja um conceito bastante amplo, não havendo uma única perspectiva que englobe todos os seus aspectos (Jerusalinsky, 2007), de maneira geral, é caracterizado por trans- 
formações em diferentes áreas da vida da criança: motora, cognitiva, social, linguagem etc. Estes processos abarcam desde aspectos ambientais, nutricionais e familiares até condições culturais (Brazelton, 2002; Maria-Mengel \& Linhares, 2007; Oliveira, Flores, \& Souza, 2012). Particularmente, a concepção psicanalítica de desenvolvimento propõe que não se perca de vista a dimensão psíquica existente na articulação com as demais funções em desenvolvimento. Ou seja, concebe que o desenvolvimento humano não opera somente a partir de automatismos biológicos, mas que essas funções orgânicas também se ordenam a partir de inscrições psíquicas imbricadas nas primeiras relações entre criança e cuidadores. Assim, trata-se de um processo constituinte, que inaugura também a criança enquanto um sujeito (Jerusalinsky, 2007, 2011).

Nessa direção, a partir de uma demanda do Ministério da Saúde, uma equipe de profissionais e especialistas em desenvolvimento infantil organizou a Pesquisa Multicêntrica de Indicadores de Risco para o Desenvolvimento Infantil. Este estudo visou a construção de um instrumento que pudesse articular o desenvolvimento e o sujeito psíquico, sem desconsiderar o processo de maturação biológica (Kupfer et al., 2009; Pesaro, 2010). Dessa forma, foi construído o instrumento Indicadores Clínicos de Risco para o Desenvolvimento Infantil (IRDI), validado para o uso de pediatras. Em sua versão original, com 31 indicadores, objetiva a identificação de risco de desenvolvimento, sem especificação diagnóstica, em crianças com idade entre 0 e 18 meses. O IRDI está organizado a partir de quatro eixos (suposição de sujeito, estabelecimento de demanda, alternância presença-ausência e função paterna) e tem seus itens divididos em quatro faixas etárias: 0 a 4 meses, 4 a 8 meses, 8 a 12 meses, e 12 a 18 meses (Kupfer et al., 2009). Os indicadores, quando presentes, são sinal de desenvolvimento saudável, e quando ausentes, indicam risco (Kupfer et al., 2009; Kupfer, Rocha, Cavalcanti, Ulhôa, \& Fingerman, 2008).

Diversas são as pesquisas já realizadas com este instrumento no Brasil. Mariotto (2016), em uma revisão bibliográfica, aponta o uso do IRDI de diferentes formas no contexto da saúde: em atividades de educação permanente e formação com profissionais de enfermagem e ACS; em interlocução com a fonoaudiologia; junto à Associação de Assistência à Criança Deficiente; em atendimento de puericultura da atenção primária; como guia para a observação no Programa da Criança; e em estudos sobre associação entre presença de risco e tipo de aleitamento. Outras publicações retrataram o uso do IRDI no contexto da educação infantil (Ferrari, Fernandes, Silva, \& Scapinello, 2017; Mariotto, 2009; Mariotto \& Bernardino, 2012; Pesaro \& Kupfer, 2016). De modo geral, esse instrumento permite uma sistematização de observações que podem sustentar a recomendação de um tratamento para bebês em risco (Lerner et al., 2013). Assim, os demais profissionais da $A B$ podem se beneficiar de seu uso, tendo em vista que a intervenção precoce está associada à evolução mais favorável desses quadros (Mariotto, 2016).

Para o melhor desenvolvimento das ações dos ACS, o Ministério da Saúde reconhece a necessidade de qualificação profissional permanente (Melo, Quintão, \& Carmo, 2015). Alguns trabalhos, como descrito anteriormente, abordam processos formativos com profissionais da $\mathrm{AB}$ para a utilização do IRDI, mais especificamente, profissionais de enfermagem e ACS. Esses estudos indicaram efeitos positivos de tias experiências formativas, no sentido de ampliar a consideração dos profissionais acerca dos aspectos psicológicos e relacionais envolvidos no desenvolvimento infantil, em comparação a momentos anteriores à formação (Morais, 2013; Tocchio, 2013).

Em relação à formação sobre o IRDI já realizada por Morais (2013) com ACS, a metodologia utilizada consistiu em quatro encontros formativos e monitoria, realizados ao longo de aproximadamente um ano. Para a avaliação da atividade, utilizou-se fichas de acompanhamento que indicaram que, dos 40 respondentes, $72,5 \%$ perceberam influência da formação sobre seu trabalho, $22,2 \%$ afirmaram um aprimoramento do conhecimento e da observação de mães/ bebês/famílias e 78,9\% indicaram intenção de continuidade do uso do IRDI em seu trabalho.

O presente estudo buscou descrever a percepção de ACS de uma região de Porto Alegre (RS) sobre uma atividade de formação cujo foco foi o desenvolvimento infantil e indicadores de risco. Diferentemente dos estudos apresentados anteriormente, na presente investigação buscou-se compreender o impacto dessa formação a partir das falas dos próprios ACS, coletadas por meio de grupos focais (GF), isto é, em momentos de discussão e reflexão grupal sobre o processo formativo realizado. Além disso, a formação oferecida foi organizada a partir 
de um curso de extensão, dividido em dois módulos bem definidos: um de caráter teórico e outro prático, visando primeiramente instrumentalizar os profissionais sobre os conceitos e promover reflexões sobre a infância para que, posteriormente, pudessem exercitá-los em observações orientadas para o uso do IRDI.

\section{Método}

Trata-se de um estudo qualitativo, de caráter exploratório-descritivo (Gray, 2012), derivado de um projeto de pesquisa mais amplo intitulado “Indicadores Clínicos de Risco para o Desenvolvimento Infantil: Formação com Agentes Comunitários de Saúde" (Esswein \& Levandowski, 2017), desenvolvido por participantes do Núcleo de Estudos em Desenvolvimento e Saúde (NEEDS) da Universidade Federal de Ciências da Saúde de Porto Alegre (UFCSPA).

Durante o ano de 2017, membros do NEEDS da UFCSPA desenvolveram um curso de extensão intitulado "Curso de Educação Continuada Sobre Indicadores Clínicos de Risco para o Desenvolvimento Infantil (IRDI)". Trata-se de uma formação sobre desenvolvimento infantil que buscou apresentar aos ACS os principais processos e etapas do desenvolvimento infantil de crianças de 0 a 18 meses e capacitá-los para a utilização do IRDI em seu contexto de trabalho.

Inicialmente, contatou-se a Gerência Distrital de Saúde de uma das regiões de Porto Alegre e foi agendada uma reunião com os coordenadores das UBS para a apresentação da proposta de pesquisa e do curso de formação. Os coordenadores que autorizaram a realização das atividades repassaram o convite aos ACS de suas equipes, nas UBS. Aqueles ACS que tiveram interesse em participar realizaram sua inscrição por meio de contato com o grupo de pesquisa.

A estrutura do curso de extensão foi organizada a partir de dois módulos: teórico, realizado nas dependências da universidade, e prático, realizado no território atendido pelos ACS. O primeiro (21h) teve como principais objetivos a sensibilização sobre a complexidade e a integralidade do desenvolvimento; a aproximação dos profissionais com as concepções de desenvolvimento infantil necessárias para compreender o IRDI; e a discussão detalhada de cada indicador do IRDI. Para tal, foram organizados sete encontros, com duração de
3 horas cada e periodicidade semanal, nos quais se utilizou como técnica a exposição oral e dialogada dos conteúdos e a realização de seminários. Todos os encontros iniciavam com uma breve retomada das temáticas trabalhadas no encontro anterior. Ainda como recursos, foram utilizados vídeos para ilustrar cada indicador do IRDI e discutir sua observação no contexto de trabalho; um material informativo sobre desenvolvimento infantil, confeccionado pelos ministrantes; um material do Ministério da Saúde sobre a atuação dos ACS, desenvolvimento e saúde mental; um manual para a observação dos itens do IRDI, adaptado de Morais (2013); e fichas confeccionadas para exercícios de observação e avaliação dos indicadores pelos ACS (Kupfer et al., 2009). Este módulo contou com a participação de 14 ACS ao todo. No decorrer dos encontros houve apenas uma desistência, atribuída à demanda de trabalho da UBS do profissional.

Nos encontros 1 e 2, foram realizadas atividades introdutórias para apresentação dos participantes, uma conversa para conhecer a percepção deles sobre a importância de seu trabalho no acompanhamento e atenção ao desenvolvimento de bebês e crianças; a construção coletiva de um conceito de desenvolvimento; uma reflexão sobre as necessidades e cuidados de que um bebê precisa; e a apresentação dos quatro eixos teóricos nos quais o IRDI está embasado. Nos encontros de 3 a 6 , foi apresentado o IRDI, seus indicadores e as características do desenvolvimento infantil de cada faixa etária avaliada. O conteúdo e os encontros foram organizados a partir das quatro faixas etárias do instrumento. Por fim, no encontro 7 foi feita uma retomada geral sobre o desenvolvimento infantil e, após, realizou-se um exercício de observação dos indicadores a partir de vídeos de interação mãe-bebê, com posterior discussão. Esta atividade oportunizou também o esclarecimento de dúvidas. Finalizou-se o encontro com um GF, a fim de conhecer a percepção dos profissionais sobre a formação até então.

Já o módulo prático (15h) objetivou dar suporte aos ACS para a incorporação dos conhecimentos trabalhados e o uso do IRDI em sua prática profissional. Este módulo contou com a participação de 9 ACS que já haviam finalizado o módulo teórico e que tiveram interesse e disponibilidade em dar continuidade à formação. Essa etapa foi estruturada a partir de três atividades, detalhadas no Quadro 1. 
Quadro 1

Descrição das atividades do módulo prático da formação oferecida aos ACS.
Atividade
Descrição

Os ACS selecionaram famílias que acompanhavam e que tivessem crianças com idade de 0 a 18 meses e pediram autorização para realizar visitas acompanhados por um dos ministrantes do curso. Nestas visitas, os ACS observaram a relação mãe-bebê a partir do IRDI, completando posteriormente a avaliação dos indicadores a partir da observação realizada. Foram realizadas visitas semanais de $1 \mathrm{~h}$ ao longo de quatro semanas, com carga horária total de $4 \mathrm{~h}$.

Após as visitas acompanhadas, foram realizadas visitas domiciliares apenas pelos ACS para observar os indicadores do IRDI, tanto em famílias

Visitas de observação não acompanhadas (apenas ACS)

Supervisão das atividades dos ACS pelos ministrantes da formação que visitariam como parte de seu trabalho de rotina quanto nos casos em que não foi possível a identificação dos indicadores nas visitas acompanhadas, realizadas previamente. Foram realizadas visitas semanais de $1 \mathrm{~h}$ ao

longo de quatro semanas, com carga horária total de $4 \mathrm{~h}$.

A partir de cada visita de observação realizada pelos ACS, organizou-se um momento de supervisão do processo para orientar, discutir e refletir sobre os aspectos observados e como se relacionavam aos indicadores de risco constantes no IRDI. Foi realizado ao menos um encontro semanal de $1 \mathrm{~h}$, ao longo de quatro semanas, totalizando $4 \mathrm{~h}$ de supervisão.
Tendo em vista as diferentes configurações de trabalho e território dos ACS, as atividades ocorreram em singulares composições, no decorrer de três meses. No entanto, algumas diretrizes orientaram o processo de forma geral: a) as visitas foram precedidas pela leitura dos itens relativos à faixa etária em observação e reflexão referente às características do desenvolvimento; b) recomendou-se que os ACS preenchessem o protocolo de observação após uma reflexão sobre a visita; c) orientou-se que a leitura realizada pelos indicadores deveria ser articulada e levar em consideração o contexto e o cotidiano da família e do território; d) qualquer caso em que se identificasse sinais de risco deveria ser reportado à equipe de saúde. Também foi realizado contato com a equipe responsável pelo matriciamento desses casos na região para a apresentação da formação e do material trabalhado, visando assegurar o suporte da rede de atenção para as situações mencionadas no item 4 , tendo em vista que esta equipe, especificamente, poderia ser acionada pelos ACS ou por sua UBS.

Ao final dessas atividades práticas, um último encontro realizado na universidade, com $3 \mathrm{~h}$ de duração, permitiu a retomada geral da formação e a revisão das instruções de observação dos indicadores do IRDI contidas no manual previamente disponibilizado pela equipe, considerando as experiências dos ACS. Nessa ocasião, também foi realizado um GF para a avaliação das atividades práticas pelos participantes e a investigação das eventuais repercussões da formação em seu trabalho.

A partir da experiência de formação, participaram deste estudo, ao total, 13 ACS atuantes em nove diferentes UBS da região norte de Porto Alegre. Trata-se de uma área que conta, no total, com aproximadamente 142 ACS (Secretaria Municipal de Saúde de Porto Alegre, 2017). O critério de inclusão foi ser ACS atuante há pelo menos um ano em uma das UBS pertencentes à Gerência Distrital de Saúde correspondente à região. Não foram adotados critérios de exclusão.

Os participantes tinham média de idade de 43 anos, com média de tempo de atuação de 6 anos na AB. Os ACS com menor tempo de atuação tinham ao menos um ano e quatro meses de experiência, e o ACS com maior tempo de experiência atuava há 21 anos nesse contexto. Ou seja, trata-se de um grupo heterogêneo, com diferentes níveis de experiência profissional. 
Todos os participantes tinham como nível de escolaridade o ensino médio completo e dois deles também o ensino superior. A maioria possuía filhos e cada ACS estava responsável, naquele momento, em média, pelo acompanhamento de 19 crianças com idades de 0 a 2 anos em seu território de atuação. No entanto, quatro ACS referiram não ter ou não saber esta informação. Apenas dois dos participantes (que estavam entre os três com maior tempo de experiência) receberam algum tipo de formação anterior sobre desenvolvimento infantil e todos indicaram ter interesse na temática.

Do total de 13 ACS, 12 estiveram presentes no primeiro momento de coleta de dados (GF realizado ao final do modelo teórico) e cinco desses também no segundo momento (GF realizado ao final do modelo prático). Apenas quatro ACS participaram dos dois momentos. Um ACS, apesar de participar de toda a formação, esteve presente somente no segundo momento de coleta de dados. As perdas evidenciadas nesse segundo momento são decorrentes de desistências de participação ou impossibilidade de comparecimento no encontro, tanto por motivos pessoais como profissionais.

Para caracterizar os participantes, foi utilizado um questionário com informações sociodemográficas e profissionais, construído pelos autores para este estudo. Para a coleta de dados sobre a percepção dos profissionais acerca da formação recebida, utilizou-se a técnica de GF, a partir de um roteiro de questões norteadoras para cada um dos dois encontros realizados, que abordavam a avaliação da formação, a relação com a prática profissional, dificuldades e perspectivas de utilização do IRDI. O GF é uma técnica de coleta de dados que reúne informações específicas de um grupo, oportuna na análise de implementação de programas e ações, que além de permitir coletar dados, promove reflexões sobre o tema discutido (Flick, 2009; Trad, 2009).

Como mencionado, foram realizados dois GF, um ao final de cada um dos módulos da formação (teórico e prático). Cada grupo teve a duração aproximada de 60 minutos. Foi feita a gravação em áudio da discussão dos participantes, mediante consentimento. Após, foi realizada a transcrição do material para análise.

\section{Análise dos dados}

Utilizou-se análise temática como método de análise dos relatos dos grupos focais. Este tipo de análise permite identificar padrões de temas dentro de um conjunto de dados qualitativos (Braun $\&$ Clarke, 2006). Nesse processo foram seguidas as orientações de Braun e Clarke (2006): a) familiarização com os dados, por meio de repetidas leituras do material de análise e realização de apontamentos iniciais; b) geração de códigos iniciais, por meio das primeiras codificações dos dados como um todo; c) busca de temas, agrupando códigos com temas em potencial; d) revisão dos temas, verificando se os temas operam bem em relação aos extratos codificados e em relação aos demais temas; e) definição e nomeação dos temas, por meio da revisão das especificidades de cada tema e a geração de seus nomes; e f) escrita do relatório, com a seleção de exemplos dos temas e análise final.

O processo de identificação dos temas foi orientado pelos dados, independentemente de uma estrutura de codificação pré-existente (Braun \& Clarke, 2006). Os temas foram construídos por um dos autores do artigo e a respectiva alocação das falas foi revisada por outro, visando minimizar o viés de interpretação.

\section{Considerações éticas}

$\mathrm{O}$ projeto do qual o presente estudo deriva seguiu as diretrizes para pesquisas com seres humanos de acordo com as Resoluções no 466 (2012) e no 510 (2016) do Conselho Nacional de Saúde. O projeto foi aprovado pelos Comitês de Ética em Pesquisa da UFCSPA (Parecer no 2.340.986) e da Secretaria Municipal de Saúde de Porto Alegre (SMS) (Parecer $\mathrm{n}^{\circ}$ 2.506.381). Todos os participantes assinaram o Termo de Consentimento Livre e Esclarecido (TCLE) e tiveram reembolso dos gastos com deslocamento até a universidade para a realização do módulo teórico. Também foi garantido seu anonimato. A formação integrou a carga horária semanal de trabalho dos ACS, não havendo qualquer prejuízo profissional para quem desistiu de participar.

\section{Resultados}

A análise temática indicou três temas centrais, cada um dos quais composto por dois subtemas, referentes à avaliação dos ACS sobre a formação. Os temas e subtemas são apresentados no mapa conceitual a seguir (Figura 1). 


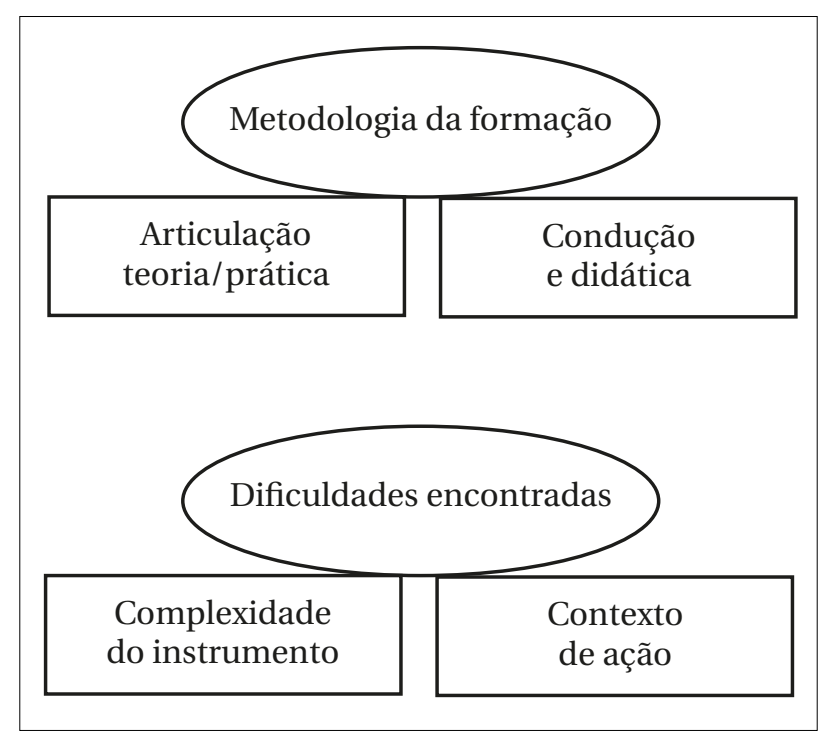

Figura 1

Mapa conceitual dos temas identificados a partir da análise temática dos relatos dos grupos focais realizados com os ACS.

\section{Metodologia da formação}

Esse tema agrupou as falas dos profissionais acerca de sua percepção sobre os aspectos da formação que referiram como importantes para a aprendizagem. Também englobou falas sobre a avaliação dos materiais utilizados, abrangendo dois subtemas: "articulação entre teoria/prática” e “condução e didática”.

Para os ACS, a articulação entre os materiais utilizados e entre os diferentes módulos foi importante para a aprendizagem. Sobre o módulo teórico, entenderam que os vídeos complementaram a teoria e o material escrito, de forma a clarear os conceitos e os indicadores apresentados, e possibilitaram melhor articulação com seu cotidiano de trabalho: "Foi bem legal porque a parte teórica tinha as explicações e depois tinha os vídeos que deixavam mais claro. Coisa que a gente não podia pegar na escrita, a gente podia pegar nos vídeos" (GF 3); “Por isso que eu acho importante a parte visual que tu colocou, que a gente, conseguindo visualizar, a gente vendo, já desperta mais o nosso dia a dia" (GF 2).

Já o módulo prático foi compreendido como o momento em que conseguiram pensar a utilização dos indicadores a partir de sua atuação. Esta articulação entre a teoria e a prática foi identificada enquanto crucial e um diferencial da proposta da formação: “Acho que foi bem legal a gente ter feito a prática pra complementar, como ela falou, unificou muito, ficou muito mais fácil' (GF 3); "Na verdade tem muitas coisas que, como tu disse na teoria, são bem sutis, né? Que às vezes precisa mais de um olhar pra conseguir enxergar, né? Mas eu acho que ficou bem claro, assim, teoria e práticas juntas, nê" (GF 3).

Dessa forma, os ACS não estabeleceram uma hierarquia entre os módulos, mas identificaram complementariedade entre eles: "Na verdade uma dependendo da outra. Só uma ou só outra acho que não seria suficiente. Com certeza a gente aprende a refinar o olhar" (GF 3).

Alguns aspectos relacionados à forma de condução dos encontros e visitas foram reconhecidos pelos ACS como facilitadores do processo de aprendizagem. A acessibilidade da linguagem do material escrito e dos encontros se mostrou importante para a compreensão dos temas e do instrumento abordado: “Não teve aquela coisa, nome da fulana e iam falar numa língua que eu, pelo menos, não ia entender. Deu pra entender do primeiro ao último" (GF 2); “Acho que foi bom, é incrível como o pessoal aqui com vários níveis, ensino médio, superior ou não, todos conseguiram absorver e gostaram do curso, conseguiu chegar até o fim, pelo menos eu acho que foi muito válido" (GF 3).

Outro aspecto mencionado pelos participantes, enquanto dificultador da aprendizagem, foi a repetição de alguns procedimentos e materiais. Entretanto, também foram identificadas falas sobre a importância dessa repetição para reforçar os aspectos trabalhados: "Achei um pouco repetitivo. Assim, metade da manhã era repetindo o que foi a semana passada, isso ficou muito repetitivo. Mas o resto foi bem bom” (GF 2); “E que nem a minha colega falou que achou muito repetitivo, de repente, por ser uma vez por semana, é melhor pra recordar" (GF 2).

\section{Dificuldades encontradas}

Esse tema reuniu as falas dos participantes relacionadas às dificuldades e obstáculos do processo formativo. Foram identificados dois subtemas: "complexidade do instrumento" e “contexto de ação".

Os participantes reconheceram algumas características do instrumento como um obstáculo durante a formação, como: quantidade de itens, diferentes níveis de dificuldade na observação dos itens para cada faixa etária e complexidade do processo de observação e identificação dos indicadores: "Eu tive dificuldade em algumas faixas etárias assim, umas que são mais difíceis de ver... Porque não é que sejam difíceis, só é bastante coisa, nê" (GF 3); “Eu notei dificuldade na parte prática entre entender o que o IRDI está falando, entender o que eu estava vendo e entender 
o que a mãe estava me passando e ao mesmo tempo falando, sabe?" (GF 3).

Algumas características do território e do contexto de trabalho também foram percebidas como barreiras para a operacionalização da formação: falta de crianças da faixa etária para acompanhamento, dificuldades de encontrar mãe e criança em momento apropriado para a visita e ausência de tempo disponível para a leitura do material: “É que eu tive bastante dificuldade em encontrar a criança mais nova acordada" (GF 3); "Minha única dificuldade é ter tempo pra leitura” (GF 2).

\section{Repercussões da formação}

Esse tema agrupou as falas dos participantes relacionadas aos desdobramentos da formação sobre a sua atividade profissional. Essas falas deram origem a dois subtemas: "mudanças de perspectiva sobre a infância" e "limitações da formação".

No primeiro deles, foi mencionada pelos participantes uma mudança na forma como percebem o desenvolvimento dos bebês. Os ACS mencionaram ter passado a compreendê-lo a partir de uma perspectiva mais ampliada e a identificar, em práticas cotidianas, aspectos do desenvolvimento que anteriormente não percebiam: "Eu acredito muito nessa coisa de apurar assim, apurar o olhar. Coisa que a gente não tinha. Esse curso abriu, independente da gente poder usar ele no nosso dia a dia do serviço, nos abriu para um outro olhar" (GF 3); "A gente tem esse olhar, parece um pouco mais sensível pra ver a criança" (GF 3).

Nessa direção, os ACS referiram incluir, em sua concepção de desenvolvimento, a dimensão do psiquismo e da saúde mental desde os primeiros meses de vida, para além dos aspectos psicomotores já reconhecidos antes da formação: "Eu nunca pensei numa avaliação assim. Eu achava que nessa idade a gente avaliasse só a parte motora mesmo, não a saúde mental" (GF 3).

Assim, foi possível perceber que os participantes produziram também um novo sentido para a atenção em saúde mental, bem como novos posicionamentos frente às intervenções: "aí a gente acaba desafogando o tubo da saúde mental, vai procurando outros caminhos..." (GF2); "Que, às vezes, pode também pode existir uma coisinha que faltou na convivência, mas não é saúde mental" (GF 2).
Porque a gente tem uma linha de taxar 'Ah, tá com problema, com dificuldade, ah, saúde mental, vamos encaminhar pro (serviço especializado), tá precisando de psicólogo, psiquiatra, alguma coisa, né. E não é bem assim. Se tu parar pra pensar, tem certas coisas que faltou o estímulo, faltou o olhar da mãe, faltou o apoio, aquele vínculo, né, que precisa, de criar ou de separar, não necessariamente a criança tem problema (GF 2).

Outra forma de conceituar a mudança do olhar sobre a infância esteve vinculada à qualificação profissional. Os ACS relataram o aprimoramento da habilidade técnica para acompanhar o desenvolvimento de bebês em seu contexto de trabalho: "Agora a gente tem um olhar mais técnico, além do dia a dia, do cotidiano, que a gente aprendeu" (GF 2); "Tu chega ali e não tá mais deduzindo, no achômetro, tu tá com um instrumento que te indica aquilo ali. Pra mim já me deu uma outra expectativa, assim, já mudou minha forma de visita. A gente vai treinando, dia a dia" (GF 2).

Este "olhar mais técnico" pareceu estar imbricado em outras possibilidades de intervenção. Nesse sentido, os ACS reportaram que o conhecimento sobre desenvolvimento e indicadores de risco pode balizar e subsidiar algumas de suas práticas.

O nenê ia ir lá e passar despercebido. Agora tu consegue constatar, já no lar da pessoa, como é o nosso papel. Tu vai lá, tu visita, tu entra no cotidiano, no ambiente da pessoa, no lar da pessoa ali, e agora tu consegue constatar com um olhar mais apurado, mais refinado, mais técnico, de percepção mais aguçada do desenvolvimento da criança (GF 2).

Assim, reconheceram a possibilidade de identificação de crianças com risco de desenvolvimento que antes passaria desapercebido e, a partir disso, a possibilidade de pensar em intervenções também junto à equipe.

... que nem a gente tava falando, talvez passaria despercebido num território todo, toda equipe tá indo na casa, mas quando tu percebe isso, tu pode trazer isso pra equipe, discutir com a equipe e pedir um auxílio de toda equipe, né, de multiprofissionais (GF 3).

Já se percebe agora, e a gente acompanha daí, né, orienta. Quando a criança volta na próxima con- 
sulta, na verdade tu já vai ter conversado com a tua equipe, ou com enfermeiro, ou com médico sobre o que tu percebeu de ausência através do instrumento (GF 2).

No que se refere à continuidade da utilização do instrumento, alguns participantes mencionaram a intenção de utilizar o IRDI em todas as visitas, enquanto outros somente para alguns casos em específico. Estes, no entanto, deram indícios de uma intenção de uso da metodologia empregada no módulo prático da formação, particularmente, o registro do que foi observado após as observações realizadas durante a visita domiciliar: "Eu vou usar ele no dia a dia né, com as minhas bebês eu vou ter que usar sempre" (GF3); "Eu acredito que vou usar esporadicamente. Eu não sei se eu vou dar conta, a gente tem muita criança, pelo amor de Deus!" (GF 3); “... depois que tu acaba a visita, tu volta pra unidade e reflete nele ali" (GF 3).

Os participantes também reconheceram algumas limitações relacionadas ao alcance das repercussões da formação em seu trabalho. Foi citado sobretudo o caráter individualizado do processo formativo, pois a forma como o conhecimento é articulado ao trabalho também depende da implicação de cada um. Esta característica foi reconhecida como um obstáculo para intervenções em equipe e junto a outros profissionais que não participaram da formação, ou mesmo que apresentaram outro nível de implicação frente à proposta: “... é uma formação pessoal para o meu trabalho. Mas, no contexto como equipe e unidade de saúde, já é outra história" (GF 3); "É, eu acho que dentro do posto se torna uma capacitação pessoal tua. De repente assim, tu, da tua parte, de cada um, porque hoje em dia é muito difícil todos né, todos num geral, mas tu se dispor" (GF 3).

Por outro lado, essa individualidade também foi reconhecida como um modo peculiar pelo qual cada ACS se apropriou da formação e do instrumento, o que está relacionado à particularidade da experiência que teve em seu território. “. . . porque aquilo que tu absorveu, todos nós olhando o IRDI e estamos na mesma sala, mesmo espaço, mas cada um absorve de uma maneira diferente do jeito de cada um e vai usaro instrumento também da sua forma" (GF 3).

\section{Discussão}

Como visto, a metodologia empregada na experiência de formação permitiu aos ACS a articulação entre teoria e prática, um aspecto importante para a consolidação de propostas de capacitação em saúde (Campos, Soares, Trapé, Silva, \& Silva, 2009; Cardoso et al., 2011; Tanaka \& Lauridsen-Ribeiro, 2009; Waidman, Costa, \& Paiano 2012). Este modelo metodológico, composto por encontros formativos e acompanhamento em prática, também esteve presente em outras formações sobre desenvolvimento e indicadores de risco, com a utilização do IRDI, no contexto da AB (Morais, 2013; Tocchio, 2013). No entanto, especificamente quanto à formação relatada e avaliada no presente estudo, os módulos estavam bem definidos e organizados em sequência.

As formações de ACS têm sido caracterizadas pela hierarquia da prática em relação à teoria (Melo et al., 2015). Contudo, é necessário compreender que, para o domínio do conhecimento empírico, também é necessário o domínio dos conceitos que orientam determinada intervenção. Dessa forma, trata-se de um equívoco pensar que exista prática sem teoria e vice-versa (Campos et al., 2009). Nessa direção, a complementaridade dos módulos, citada pelos ACS enquanto uma exceção dentre as formações de que costumam participar, indica um possível avanço em relação à hierarquização teoria/prática, o que repercute na forma como se relacionam com o objeto de conhecimento. Enquanto o módulo teórico permitiu a discussão de conceitos de desenvolvimento infantil necessários para o módulo prático, a experiência in loco possibilitou a ilustração e a descoberta de caminhos possíveis para a utilização do IRDI em seu trabalho.

O subtema "complexidade do instrumento" indica que os participantes tiveram dificuldades frente a algumas características do IRDI, como a complexidade do processo de observação. Outros estudos já apontaram dificuldades na utilização do IRDI para a observação de bebês, tendo em vista que alguns itens estão relacionados a momentos específicos de interação mãe-bebê, por exemplo, alimentação (item 22) ou cuidados corporais (item 15) (Arpini, Zanatta, Marchesan, Savegnago, \& Bernardi, 2015). No entanto, é importante destacar que os ACS estão acostumados a utilizar instrumentos de rápido preenchimento, que não dependem de uma interpretação, o que poderia levar ao preenchimento do IRDI como um questionário (Morais, 2013). Em relação a isso, Lerner et al. (2013) destacaram que privilegiar os aspectos relacionais existentes entre criança e cuidadores no desenvolvi- 
mento da criança faz com que o IRDI não se reduza ao preenchimento de mais uma escala. Nesta direção, a metodologia da formação deste estudo priorizou a interpretação dos indicadores sobre a observação, o que pode explicar, em parte, a dificuldade referida pelos participantes. Assim, essa metodologia pareceu promover uma "desacomodação" dos ACS em relação ao modo corriqueiro de trabalho.

No entanto, algumas falas dos profissionais ainda sugeriram a tentativa de "decorar" o instrumento para sua melhor utilização, o que indica a percepção de que conhecer cada item é visto como necessário. Sendo assim, considera-se que a complexidade do instrumento tenha oportunizado a necessidade de maior reflexão sobre os indicadores e os processos a serem observados, sem, no entanto, inviabilizar a utilização.

Waidman et al. (2012) apontam que os ACS podem não reconhecer e incluir em sua prática as ações de cuidado psíquico por considerarem que outras atividades têm maior prioridade. Sousa (2007) também descreveu que os ACS relacionam problemas de desenvolvimento a sofrimento psíquico, mas que, no entanto, tem dificuldades em realizar relatos dessas experiências com a população infantil. Para Sinibaldi (2013), tal dificuldade está relacionada à construção da lógica dos especialismos, que credita essas intervenções somente aos profissionais especializados. Os ACS participantes deste estudo também reconheceram falta de capacitação e formação para a execução de seu trabalho de forma geral, o que permitiu entender que, muitas vezes, suas experiências pessoais e profissionais eram a bagagem que dispunham para embasar as intervenções. Esse aspecto converge com a literatura, que indica que a formação ainda parece ser um grande impasse da $\mathrm{AB}$ em relação ao tema da saúde psíquica (Peixoto et al., 2017; Silveira et al., 2016; Tanaka \& Lauridsen-Ribeiro, 2009; Tszesnioski, Nobrega, Lima, \& Facundes, 2015; Waidman et al., 2012).

No entanto, os resultados deste estudo mostram importantes repercussões e avanços na forma como os ACS percebem seu trabalho em relação à infância a partir da formação. Novas formas de olhar e intervir nesse contexto foram identificadas a partir do subtema "mudanças de perspectiva sobre a infância”. Trata-se de uma ampliação sobre o conceito de desenvolvimento, que passa a abranger também os aspectos psíquicos e relacionais, tal como a proposta do IRDI, que articula o desenvolvimento psíquico aos processos de maturação (Kupfer et al., 2009; Pesaro, 2010). Este olhar se aproxima da integralidade do cuidado, contemplada pela PNAB (Ministério da Saúde, 2017). Nessa direção, tal definição também ajudou os ACS a pensarem sobre seu próprio trabalho e estratégias de intervenção em articulação com a rede de assistência psicossocial.

O tema também ofereceu algumas pistas sobre a forma como os ACS incorporaram ou pretendiam utilizar o IRDI em seu cotidiano, bem como os conhecimentos construídos na formação. Primeiramente, é preciso considerar que os ACS avaliaram, a partir da formação, que conseguem identificar possíveis casos de risco para o desenvolvimento. Além disso, passaram a reconhecer esta atividade enquanto parte de seu trabalho e a entender a utilização do IRDI enquanto instrumento de acompanhamento e de possível articulação com a equipe.

Destaca-se que a estratégia de formação desenvolvida foi pensada inicialmente para que os ACS não ficassem engessados nos indicadores durante a visita, mas pudessem incorporá-los em seu olhar, para posteriormente refletir e preencher o instrumento. Neste sentido, a proposta não objetivava atribuir mais uma função aos ACS, mas nomear e sugerir parâmetros para a prática de acompanhamento, que é inerente ao seu trabalho (Pesaro \& Kupfer, 2016). Sendo assim, a mudança de perspectiva sobre a infância parece ter uma repercussão maior do que o próprio uso do instrumento, uma vez que pode estar presente em todas as intervenções realizadas por esses profissionais.

Ainda, é importante ressaltar que a formação não buscou definir um curso de desenvolvimento normativo para a infância, mas salientar a importância de estar atento a alguns processos esperados em momentos específicos dessa época da vida que, quando não ocorrem, despertam um sinal de alerta (Pesaro \& Kupfer, 2016) ou, na metáfora construída pelos ACS, "acendem uma luz vermelha". Nessa direção, a articulação dos indicadores pode operar como uma leitura da tendência do desenvolvimento, de forma que possibilita identificar casos em risco, sem desconsiderar a singularidade no desenrolar dos tempos maturativos fisiológico e psíquico (Pesaro, 2010; Pesaro \& Kupfer, 2016). Assim, este alerta pode ser identificado enquanto um marcador que sugere a necessidade de acompanhamento mais próximo, bem como de maior 
esforço das equipes em intervir com a família (Santos \& Celeri, 2017). A leitura integral vai ao encontro do que é proposto pela PNAB (Ministério da Saúde, 2017) e pela política de humanização (Ministério da Saúde, 2013b; Silva, Oliveira, \& Pereira, 2015), isto é, uma perspectiva de acolhimento pautada pela via da singularidade. Considera-se que a proximidade que os ACS têm com as famílias seja uma característica propícia para esse tipo de intervenção.

Os ACS ainda comentaram o caráter individualizado da formação no subtema sobre limitações. De fato, o curso não visou a implementação do IRDI nessa região de Porto Alegre, até mesmo porque contou com um número restrito de participantes em comparação ao total de profissionais que atuam nessa gerência de saúde. Segundo os participantes, isso pode dificultar a interlocução com as equipes. Por outro lado, a individualização também pode ser interpretada como a forma singular com que cada ACS reconhece e se apropria dos indicadores, a partir de sua implicação pessoal. Percebeu-se, durante o processo formativo e nos relatos dos ACS, a existência de diferentes apropriações do instrumento e da formação como um todo. Essas particularidades estão associadas à multiplicidade de percursos de formação, que, por sua vez, vincula-se tanto ao contexto de trabalho, que envolve o território, particularidades das atividades desenvolvidas pelos ACS em sua microárea e população, quanto às características pessoais desses profissionais, experiências, implicações e disponibilidade para a formação.

Essas formas particulares de apropriação do IRDI também foram descritas em outra formação realizada com ACS e identificadas como característica potencializadora dos efeitos de permanência da formação, tendo em vista que se liga a um traço particular do sujeito (Morais, 2013). Corroboram este entendimento as falas dos ACS coletadas neste estudo, relacionadas ao subtema "Mudanças de concepção sobre a infância", que descreveram mudanças para além da qualificação de trabalho, incluindo alterações pessoais na forma como observam e compreendem o desenvolvimento infantil.

\section{Considerações finais}

Este estudo teve como objetivo descrever a avaliação de ACS de Porto Alegre sobre uma formação referente ao desenvolvimento infantil e indicadores de risco. Os resultados apontam que a metodologia empregada na formação forneceu recursos que permitiram articular a teoria e a prática, consolidando um processo de apreensão do conteúdo da formação e de incorporação dos indicadores de risco no trabalho dos ACS. Foi referida uma ampliação na concepção de desenvolvimento, sobretudo relacionada aos aspectos psíquicos. Tal mudança de olhar sobre a infância pareceu ser uma das principais contribuições da formação, uma vez que pode se fazer presente em todas as intervenções realizadas pelos ACS para este público.

Os participantes apontaram a intenção de utilizar o IRDI no acompanhamento de crianças em seu território. Em paralelo, fizeram referência à melhor qualificação de sua observação por passarem a utilizar esses indicadores enquanto parâmetros de desenvolvimento, sendo que antes consideravam apenas suas vivências pessoais e experiências profissionais.

Embora os achados sejam relevantes quando se pensa na integração ensino-pesquisa-extensão-assistência e na avaliação de intervenções, a formação e a pesquisa aqui apresentadas têm algumas limitações. Como apontado pelos ACS, a formação abrangeu um número limitado de profissionais de apenas uma região de um único município. Identifica-se também a necessidade de um tempo maior de acompanhamento in loco para avaliação dos efeitos da formação a longo prazo. No presente estudo, optou-se por um intervalo de tempo mais restrito em função do contexto de trabalho dos participantes.

Outra limitação foi o pequeno número de participantes do último GF em comparação ao anterior, em virtude de situações pessoais e profissionais que impediram o comparecimento dos ACS. Por fim, para estudos futuros, sugere-se a utilização de técnicas padronizadas e quantitativas para verificar em que medida realmente os ACS conseguiram, a partir da formação, identificar sinais de risco de desenvolvimento nas crianças dentro das faixas etárias do IRDI que acompanharam.

Mesmo com tais limitações, de forma geral, identificou-se a possibilidade de articulação do IRDI com estratégias de cuidado na $\mathrm{AB}$, sobretudo em relação ao acompanhamento do desenvolvimento infantil realizado pelos ACS. Reitera-se, nesse sentido, a importância do olhar sobre a infância que contemple também os aspectos psíquicos do desenvolvimento para refletir sobre as intervenções que abarquem o sujeito em sua integralidade, tal como é preconizado pelo SUS. 


\section{Referências}

Arpini, D. M., Zanatta, E., Marchesan, R. Q., Savegnago, S. D. O., \& Bernardi, P. H. (2015). Intervenções precoces na infância: Observando a relação mãe-bebê em um serviço de saúde. Psicologia em Revista, 21(1), 3750. http:// dx.doi.org/DOI-10.5752/P.1678-9523.2015V21N1P37

Bachilli, R. G., Scavassa, A. J., \& Spiri, W. C. (2008). A identidade do agente comunitário de saúde: Uma abordagem fenomenológica. Ciência \& Saúde Coletiva, 13(1), 5160. http://dx.doi.org/10.1590/S1413-81232008000100010

Braun, V., \& Clarke, V. (2006). Using thematic analysis in psychology. Qualitative research in psychology, 3(2), 77101. http://dx.doi.org/10.1191/1478088706qp063oa

Brazelton, N. T. B. (2002). Momentos decisivos do desenvolvimento infantil. Martins Fontes.

Campos, C. M. S., Soares C. B., Trapé C. A., Silva B. R. B., \& Silva, T. C. (2009). Articulação teoria-prática e processo ensino-aprendizagem em uma disciplina de enfermagem em saúde coletiva. Revista da Escola de Enfermagem da USP, 43(spe. 2), 12261231. http://dx.doi.org/10.1590/S0080-62342009000600014

Cardoso, F. A., Cordeiro, V. R. N., Lima D. B., Melo, B. C., Menezes, R. N. B., Moulaz, A. L. S., Sá, G. B., \& Souza, A. V. F. (2011). Capacitação de agentes comunitários de saúde: Experiência de ensino e prática com alunos de Enfermagem. Revista Brasileira de Enfermagem, 64(5), 968973. http://dx.doi.org/10.1590/S0034-71672011000500026

Ferrari, A. G., Fernandes, P. P., Silva, M. R., \& Scapinello, M. (2017). A experiência com a Metodologia IRDI em creches: Pré-venir um sujeito. Revista Latinoamericana de Psicopatologia Fundamental, 20(1), 1733. http:// dx.doi.org/10.1590/1415-4714.2017v20n1p17.2

Flick, U. (2009). Introdução à pesquisa qualitativa. Artmed.

Gray, D. (2012). Pesquisa no mundo real. Penso.

Jerusalinsky, A. (2007). Psicanálise e desenvolvimento infantil: Um enfoque transdisciplinar (6a ed.). Artes e Ofícios.

Jerusalinsky, J. (2011). A criação da criança: Brincar, gozo e fala entre a mãe e o bebê. Ágalma.

Kupfer, M. C. M., Jerusalinsky, A. N., Bernardino, L. M. F., Wanderley, D., Rocha, P. S. B., Molina, S. E., Sales, L. M., Stellin, R., Pesaro, M. E., \& Lerner, R. (2009). Valor preditivo de indicadores clínicos de risco para o desenvolvimento infantil: Um estudo a partir da teoria psicanalítica. Revista Latinoamericana de Psicopatologia Fundamental, 6(1), 4868. https://doi.org/10.1590/S1415-47142010000100003

Kupfer, M. C. M., Rocha, P. S., Cavalcanti, A. E., Ulhôa, A. M., \& Fingerman, D. (2008). Apresentação e debate em torno da pesquisa multicêntrica de indicadores clínicos de risco para o desenvolvimento infantil. In R. Lerner, \& M. C. M. Kupfer (Orgs.), Psicanálise com crianças: Clínica e pesquisa (pp. 4962). Fapesp, \& Escuta.

Lerner, R., Paolo, A. F., Campana, N. T. C., Morais, A. S., Tocchio, A. B., \& Silva, R. S. F. (2013). A psicologia na articulação entre os âmbitos coletivo e psíquico: Construção de uma política pública em saúde de cuidado com o desenvolvimento. Psicologia Ciência e Profissão, 33(n. spe.), 100111.

Maria-Mengel, M. R. S., \& Linhares, M. B. M. (2007). Fatores de risco para problemas de desenvolvimento infantil. RevistaLatino-AmericanadeEnfermagem, 15(spe.), 837842.http://dx.doi.org/10.1590/S0104-11692007000700019

Mariotto, R. M. M. (2009). Cuidar, educar e prevenir: As funções da creche na subjetivação de bebês. Escuta.

Mariotto, R. M. (2016). O instrumento IRDI no campo da saúde: Aplicações, implicações e reflexões. In M. Kamers, H. H. Marcon, \& M. L. T. Moretto (Orgs.), Desafios atuais das práticas em hospitais e nas instituições de saúde (pp. 183202). Escuta.

Mariotto, R. M. M., \& Bernardino, L. M. F. (2012). Detecção, prevenção e tratamento de riscos psíquicos precoces: Desfecho de um programa acadêmico. Psicologia Argumento, 30(71), 711717.http://dx.doi.org/10.7213/psicol.argum.7477

Melo, M. B., Quintão, A. F., \& Carmo, R. F. (2015). O programa de qualificação e desenvolvimento do agente comunitário de saúde na perspectiva dos diversos sujeitos envolvidos na atenção primária em saúde. Saúde e Sociedade, 24(1), 8699. http://dx.doi.org/10.1590/S0104-12902015000100007

Ministério da Saúde. (2006). Política Nacional de Atenção Básica. https://bit.ly/3xn7rpt

Ministério da Saúde. (2009a). O trabalho do Agente Comunitário de Saúde. https://bit.ly/2SOyn2t

Ministério da Saúde. (2009b). Guia prático do Agente Comunitário de Saúde. https:/ /bit.ly/3dMaDDj 
Esswein, G. C., Rovaris, A. F., Rocha, G. P., \& Levandowski, D. C. (2021). Percepção de Agentes Comunitários de Saúde.

Ministério da Saúde. (2012). Política Nacional de Atenção Básica. https://bit.ly/3wnf5yF

Ministério da Saúde. (2013a). Cadernos de Atenção Básica no 34 Saúde Mental.https://bit.ly/3dFLk5V

Ministério da Saúde. (2013b). Política nacional de humanização. https://bit.ly/3jL2JOc

Ministério da Saúde. (2017, 21 de setembro). Portaria nº 2.436: Aprova a Política Nacional de Atenção Básica, estabelecendo a revisão de diretrizes para a organização da Atenção Básica, no âmbito do Sistema Único de Saúde (SUS). https://bit.ly/3hjroIo

Morais, A. S. (2013). Usos e apropriações de indicadores clínicos de risco para o desenvolvimento infantil por agentes comunitários de saúde: Uma experiência de formação [Dissertação de Mestrado, Universidade de São Paulo]. Biblioteca Digital USP. https://bit.ly/3qL7g4O

Morosini, M.V., Corbo, A. D., \& Guimarães, C. C. (2007). O agente comunitário de saúde no âmbito das políticas voltadas para a atenção básica: concepções do trabalho e da formação profissional. Trabalho, Educação e Saúde, 5(2), 261280. http://dx.doi.org/10.1590/S1981-77462007000200005

Oliveira, L. D., Flores, M. R., \& Souza, A. P. R. (2012). Fatores de risco psíquico ao desenvolvimento infantil: Implicações para a fonoaudiologia. Revista CEFAC, 14(2), 333342. https://doi.org/10.1590/S1516-18462011005000054

Peixoto, J., Meira, S. S., Santos, M. R. C., Sales A. S., Casotti, C. A., \&Vilela, A. B. A. (2017). Dispositivos de cuidado em saúde mental: Percepção de agentes comunitários de saúde na Bahia-Brasil [Trabalho apresentado]. 6 Congresso Ibero-Americano em Investigação Qualitativa, Salamanca. https://bit.ly/3hA0xqc

Pesaro, M. E. (2010). Alcance e limites teórico-metodológicos da pesquisa multicêntrica de indicadores clínicos de risco para o desenvolvimento infantil [Tese de Doutorado, Universidade de São Paulo]. Biblioteca Digital USP. https://bit.ly/3hvmsik

Pesaro, M. E., \& Kupfer, M. C. M. (2016). Um lugar para o sujeito-criança: Os indicadores clínicos de risco para o desenvolvimento infantil (IRDI) como mediadores do olhar interdisciplinar sobre os bebês. Analytica: Revista de Psicanálise, 5(9), 5868. https://bit.ly/3hFeCT8

Riquinho, D. L., Pellini, T. V., Ramos, D. T., Silveira, M. R., \& Santos, V. C. F. (2017). O cotidiano de trabalho do agente comunitário de saúde: Entre a dificuldade e a potência. Trabalho, Educação e Saúde, 16(1), 163182. http://dx.doi.org/10.1590/1981-7746-sol00086

Santos, R. G. H., \& Celeri, E. H. R. V. (2017). Rastreamento de problemas de saúde mental em crianças pré- escolares no contexto da atenção básica à saúde. Revista Paulista de Pediatria, 36(1), 8290. http:// dx.doi.org/10.1590/1984-0462/;2018;36;1;00009

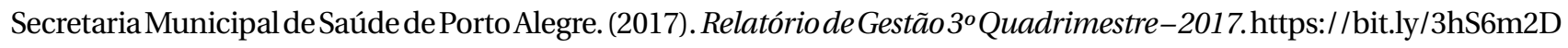

Silva, M. C. R. A. S., Oliveira, D. C., \& Pereira, E. R. (2015). A produção discursiva dos profissionais acerca da humanização em saúde: Singularidade, direito e ética. Revista Latino-americana de Enfermagem, 23(5), 936944. http:// dx.doi.org/10.1590/0104-1169.0505.2634

Silveira, T. B., Oliveira, A. M. N., Algeri, S., Susin, L. R. O., Baisch, A. L. M., Marques, L. A., \& Silva, P. A. (2016). A invisibilidade da violência psicológica pelos profissionais de saúde. Revista Brasileira de Crescimento e Desenvolvimento Humano, 26(3), 345351. http://dx.doi.org/10.7322/jhgd.122818

Sinibaldi, B. (2013). Saúde mental infantil e atenção primária: Relações possíveis. Revista de Psicologia da UNESP, 12(2), 6172. https://bit.ly/3huy2Ko

Sousa, G. C. (2007). O agente comunitário de saúde e a saúde mental: Percepções e ações na atenção às pessoas em sofrimento mental [Dissertação de Mestrado, Universidade de São Paulo]. Biblioteca Digital da USP. https://bit.ly/3qL7FUS

Tanaka, O.Y., \& Lauridsen-Ribeiro, E. (2009). Ações de saúde mental na atenção básica: Caminho para ampliação da integralidade da atenção. Ciência \& Saúde Coletiva, 14(2), 477486. http://dx.doi.org/10.1590/S1413-81232009000200016

Tocchio, A. B. (2013). Educação permanente de profissionais de enfermagem da atenção básica à saúde a partir de indicadores clínicos de risco para o desenvolvimento infantil. [Dissertação de Mestrado, Universidade de São Paulo]. Biblioteca Digital USP. https://bit.ly/3qLoWNP

Trad, L. A. B. (2009). Grupos focais: Conceitos, procedimentos e reflexões baseadas em experiências com o uso da técnica em pesquisas de saúde. Physis, 19(3), 777796. http://dx.doi.org/10.1590/\$0103-73312009000300013 
Tszesnioski, L. C., Nobrega, K. B. G., Lima, M. L. L. T., \& Facundes, V. L. D. (2015). Construindo a rede de cuidados em saúde mental infantojuvenil: Intervenções no território. Ciência e Saúde Coletiva, 20 (2), 363370. http://dx.doi.org/10.1590/1413-81232015202.05082014

Waidman, M.A. P., Costa, B., \& Paiano, M. (2012). Percepções e atuação do agente comunitário de saúde em saúde mental. Revista da Escola de Enfermagem da USP, 46(5), 11701177. http://dx.doi.org/10.1590/S0080-62342012000500019

\section{Georgius Cardoso Esswein}

Mestre em Psicologia e Saúde pela Universidade Federal de Ciências da Saúde de Porto Alegre (UFCSPA), Porto Alegre - RS. Brasil. Doutorando em Psicologia pela Universidade Federal do Rio Grande do Sul (UFRGS), Porto Alegre - RS. Brasil. Bolsista de doutorado do CNPq.

E-mail: georgius.esswein@gmail.com

(1) https://orcid.org/0000-0002-6757-1954

\section{Aline Facchin Rovaris}

Psicóloga do Centro de Atenção Psicossocial (CAPS) Novos Tempos, Canoas - RS. Brasil.

E-mail: alinefacchinrovaris@gmail.com

(1) https://orcid.org/0000-0001-8608-2112

Gabrielli Pohlmann Rocha

Graduada em Psicologia pela Universidade Federal de Ciência da Saúde de Porto Alegre (UFCSPA), Porto Alegre RS. Brasil. Residente do Programa de Residência Multiprofissional em Saúde Mental pela Universidade Estadual de Campinas (Unicamp), Campinas - SP.

E-mail: gabriellipr@gmail.com

(1) https://orcid.org/0000-0001-9976-2993

\section{Daniela Centenaro Levandowski}

Professora Associada do Departamento de Psicologia e dos programas de pós-graduação em Psicologia e Saúde e em Ciências da Saúde da Universidade Federal de Ciência da Saúde de Porto Alegre (UFCSPA), Porto Alegre - RS. Brasil. Bolsista Produtividade em Pesquisa do CNPq.

E-mail: danielal@ufcspa.edu.br

(1) https://orcid.org/0000-0002-6338-7287

Endereço de correspondência:

Rua Sarmento Leite, 245, Sala 207, Prédio 1, Centro Histórico. CEP: 90050-170. Porto Alegre - RS. Brasil.

Recebido 20/11/2018

Aceito 07/01/2020

Received 11/20/2018

Approved 01/07/2020

Recibido 20/11/2018

Aceptado 07/01/2020 
Esswein, G. C., Rovaris, A. F., Rocha, G. P., \& Levandowski, D. C. (2021). Percepção de Agentes Comunitários de Saúde.

Como citar: Esswein, G. C., Rovaris, A. F., Rocha, G. P., \& Levandowski, D. C. (2021). Percepção de agentes comunitários de saúde sobre uma formação em desenvolvimento infantil e indicadores de risco. Psicologia: Ciência e Profissão, 41 (n.spe 4), 1-15. https://doi.org/10.1590/1982-3703003216196

How to cite: Esswein, G. C., Rovaris, A. F., Rocha, G. P., \& Levandowski, D. C. (2021). Perceptions of community health agents about a course on child development and risk indicators. Psicologia: Ciência e Profissão, 41 (n.spe 4), 1-15. https://doi.org/10.1590/1982-3703003216196

Cómo citar: Esswein, G. C., Rovaris, A. F., Rocha, G. P., \& Levandowski, D. C. (2021). Percepción de agentes comunitarios de salud sobre la capacitación en desarrollo infantil e indicadores de riesgo. Psicologia: Ciência e Profissão, 41 (n.spe 4), 1-15. https:// doi.org/10.1590/1982-3703003216196 